\title{
Uso de lodo de esgoto na produção e na qualidade de mudas e teores de nutrientes em Hovenia dulcis (Rhamnaceae)
}

\author{
Henrique Souto Melo' ${ }^{(1)}$, Ana Caroline Machado Gonçalves' ${ }^{1}$, \\ Amanda Oliveira Travessas ${ }^{2}$ (i) \& Silvane Vestena ${ }^{1, *}$ (i)
}

\author{
Universidade Federal do Pampa - Campus São Gabriel, Rua Aluízio Barros Macedo, s/n, BR 290, - Km 423, 97300-970, \\ São Gabriel, Rio Grande do Sul, Brasil. \\ 2Programa de Pós-graduação em Biologia de Ambientes Aquáticos Continentais, Instituto de Ciências Biológicas, Universidade Federal \\ do Rio Grande - FURG, Av. Itália, Km 8 - Campus Carreiros, CEP: 96203-900, Rio Grande, Rio Grande do Sul, Brasil. \\ Autor para correspondência: silvanevestena@gmail.com
}

Recebido em 17.IX.2019

Aceito em 03.V.2021

DOI 10.21826/2446-82312021v76e2021020

\begin{abstract}
RESUMO - O objetivo do estudo foi avaliar o efeito de diferentes substratos contendo lodo de esgoto na qualidade e no teor de macro e micronutrientes em Hovenia dulcis Thunberg. Os tratamentos consistiram em diferentes composições de substrato contendo lodo de esgoto, sendo mensuradas as características biométricas e, determinado os teores de macro e micronutrientes. Observou-se que a ordem crescente do teor total dos macronutrientes avaliados na raiz foi de $\mathrm{K}>\mathrm{N}>\mathrm{Mg}>\mathrm{Ca}>\mathrm{P}>\mathrm{S}$ e na parte aérea $\mathrm{Ca}>\mathrm{N}>\mathrm{K}>\mathrm{P}>\mathrm{Mg}>\mathrm{S}$; para os micronutrientes, o teor na raiz foi de $\mathrm{Fe}>\mathrm{Zn}$ $>\mathrm{Mn}>\mathrm{B}>\mathrm{Cu}$ e na parte aérea $\mathrm{Fe}>\mathrm{Mn}>\mathrm{Zn}>\mathrm{B}>\mathrm{Cu}$. O lodo de esgoto como substrato proporcionou mudas de melhor qualidade nutricional, com melhor crescimento e aumento de biomassa aérea e radicular.
\end{abstract}

Palavras-chave: características morfológicas, nutrientes minerais

ABSTRACT - Use of sewage sludge in production, seedling quality and nutrient content in Hovenia dulcis (Rhamnaceae). The objective of this study was to evaluate the effect of different substrates containing sewage sludge on the quality and content of macro and micronutrients of Hovenia dulcis Thunberg. The treatments were composed of different substrate compositions containing sludge and sewage, and biometric characteristics were measured and macro and micronutrient contents were determined. It was observed that the increasing order of the total macronutrient content evaluated in the root was $\mathrm{K}>\mathrm{N}>\mathrm{Mg}>\mathrm{Ca}>\mathrm{P}>\mathrm{S}$ and at the aerial part $>\mathrm{Ca}>\mathrm{N}>\mathrm{K}>\mathrm{P}>\mathrm{Mg}>\mathrm{S}$; for the micronutrients, at the root content was $\mathrm{Fe}>\mathrm{Zn}>\mathrm{Mn}>\mathrm{B}>\mathrm{Cu}$ and in the aerial part $\mathrm{Fe}>\mathrm{Mn}>\mathrm{Zn}>\mathrm{B}>\mathrm{Cu}$. The use of sewage sludge as substrate provided better nutritional quality seedlings with better growth and increase of aerial and root biomass.

Keywords: morphological characteristics, mineral nutrients

\section{INTRODUÇÃO}

O Brasil possui uma crescente demanda por produtos de origem florestal, implicando no aumento do consumo de fertilizantes na produção de mudas e na ocasião de plantio (Caldeira et al. 2012). Com base nisso, a utilização de componentes orgânicos, tal como o lodo de esgoto, a casca de arroz carbonizada, a fibra de coco, o esterco bovino, a cama de frango, o esterco de codorna, dentre outros, podem contribuir para redução do uso de fertilizantes minerais. Além disso, quando utilizado como substrato para a produção de mudas, se torna uma alternativa para a redução de custos, tendo em vista sua disponibilidade $\mathrm{e}$ facilidade de aquisição (Trazzi et al. 2014).

Vários fatores devem ser considerados para se obter florestas com alto padrão de qualidade, sendo um dos principais a qualidade das mudas utilizadas no plantio, que devem apresentar adequada nutrição, serem vigorosas, resistentes ao estresse do transplante e livre de pragas e doenças, assegurando boa adaptação e crescimento após o plantio (Cruz et al. 2004). A obtenção de mudas de boa qualidade exige a utilização de substrato que forneça os nutrientes necessários ao pleno desenvolvimento da planta, pois o substrato exerce uma influência marcante na arquitetura do sistema radicular e no estado nutricional das plantas, afetando a qualidade das mudas (Trazzi et al. 2014).

A utilização de resíduos orgânicos urbanos na composição de substratos é uma alternativa que pode ser viável na produção de mudas, como é o caso do lodo de esgoto urbano, cujo material é composto basicamente de matéria orgânica (Santos et al. 2013, Gonçalves et al. 2014). Este resíduo pode ser usado como condicionador das propriedades físicas, químicas e biológicas do solo, considerando seu alto teor de matéria orgânica e de nutrientes (Prates et al. 2011, Ferraz et al. 2016). 
Segundo Paiva et al. (2009), Scheer et al. (2010) e Gonçalves et al. (2014) a disposição final do lodo de esgoto vem se caracterizando como um dos problemas ambientais urbanos mais relevantes da atualidade e, que cresce diariamente, reflexo da ampliação das redes de coleta e incremento dos níveis de tratamento. Nos países mais desenvolvidos o lodo de esgoto vem sendo utilizado na agricultura e nos plantios florestais como fonte de matéria orgânica, de macro e de micronutrientes e, como condicionador de solos pobres e desestruturados (Paiva et al. 2009, Ferraz et al. 2016).

Considerando o problema da destinação do lodo esgoto de estações de tratamento, a busca de novas formulações de substratos que diminuam os custos de produção e a crescente degradação ambiental dos Biomas Brasileiros, a utilização desses substratos alternativos na produção de mudas de espécies nativas e/ou exóticas para a recuperação de áreas degradadas e/ou para estabelecimento de novas florestas para recuperação da biodiversidade de espécies é de grande relevância.

A uva-do-japão (Hovenia dulcis), pertencente da família Rhamnaceae, é uma espécie arbórea exótica com grande habilidade adaptativa, sendo bastante utilizada para recuperação de áreas degradadas, atração da fauna (aves e mamíferos) pela grande quantidade de frutos produzidos, reflorestamento ciliar de açudes e barragens, cultivada para fins de ornamentação e como barreira de vento (Wandscheer et al. 2011, Zhou et al. 2013). Cabe ressaltar que, a espécie em estudo é exótica e, apontada por alguns pesquisadores como uma das maiores invasoras de florestas nativas brasileiras; entretanto, quando se pensa em implantação de plantios comerciais, recuperação de áreas degradadas ou recomposição de florestas, ou mesmo para atrativo da fauna e/ou alimentação animal, pode ser considerada como espécie promissora (Rosa et al. 2008, Noernberg 2009, Ribeiro et al. 2019).

Diante do exposto, este estudo teve como objetivo avaliar o potencial do lodo de esgoto como substrato na produção de mudas de Hovenia dulcis e determinar o teor de micro e macronutrientes nas raízes e parte aérea das mudas.

\section{MATERIAL E MÉTODOS}

O experimento foi realizado na Universidade Federal do Pampa (UNIPAMPA) - Campus São Gabriel (30²0'11" S e 54 ${ }^{\circ} 19^{\prime} 11^{\prime}$ 'W, 114 m de altitude), município de São Gabriel, Rio Grande do Sul; conduzido em casa de vegetação, coberta com polietileno de baixa densidade (PeBD) de $100 \mu \mathrm{m}$, sombrite de $50 \%$.

Os frutos de $H$. dulcis foram coletados no Município de São Gabriel, RS. Posteriormente os mesmos foram conduzidos ao Laboratório de Botânica da UNIPAMPA, onde foram despolpados manualmente, macerados e lavados com água corrente com auxílio de peneira, de modo a separar as sementes da polpa. As sementes foram, então, colocadas para secar a sombra e sobre papel filtro, eliminando-se as sementes imaturas, deterioradas ou danificadas por insetos.
O lodo utilizado foi obtido da Estação de Tratamento de Esgoto São Gabriel Saneamento, São Gabriel, RS, sendo que o lodo de esgoto foi previamente higienizado pelo processo de solarização, durante 40 dias. Este processo resulta na produção de um biossólido de melhor perfil sanitário, com o intuito de promover uma prévia desinfecção e desinfestação de patógenos e, consequentemente, menores restrições para o uso agrícola (Faustino et al. 2005, Caldeira et al. 2014).

Para a composição dos tratamentos foram utilizados três materiais: substrato comercial Plantmax ${ }^{\circledR}(\mathrm{SC})$, cama de equino semidecomposta (CA) e lodo de esgoto (LE). Os substratos foram combinados da seguinte forma: T1 $(50 \%$ $\mathrm{SC}+50 \% \mathrm{CA})$, denominado composto orgânico (CO), T2 (20\% LE + 80\% CO), T3 (40\% LE + 60\% CO) e $\mathrm{T} 4(60 \% \mathrm{LE}+40 \% \mathrm{CO})$. Por sua vez, o substrato T1, isento de lodo de esgoto, foi utilizado como testemunha. Após a composição dos tratamentos foram avaliados os teores dos nutrientes cálcio $(\mathrm{Ca})$, magnésio $(\mathrm{Mg})$, fósforo $(\mathrm{P})$, potássio $(\mathrm{K})$, zinco $(\mathrm{Zn})$, cobre $(\mathrm{Cu})$, enxofre $(\mathrm{S})$, boro (B), ferro $(\mathrm{Fe})$, manganês $(\mathrm{Mn})$, sódio $(\mathrm{Na})$ e nitrogênio (N), sendo as análises realizadas no Laboratório de Solos da Universidade Federal de Santa Maria (UFSM), Santa Maria, RS.

A semeadura foi realizada em tubetes de polietileno de $200 \mathrm{~cm}^{3}$ cada, contendo uma semente por tubete, dispostas em bancadas metálicas a $100 \mathrm{~cm}$ de altura do solo. A irrigação foi realizada diariamente por sistema automático de irrigação por microaspersão, visando manter a umidade dos substratos, contribuindo à germinação e, posteriormente emergência das plântulas.

O experimento foi arranjado no delineamento inteiramente casualizado, composto por quatro tratamentos (T1, T2, T3 e T4) com seis repetições (bandejas) para cada tratamento e 30 células cada bandeja. As médias dos tratamentos foram submetidas à análise de variância e, quando observadas diferenças significativas pelo teste F, foram comparadas pelo teste Tukey ao nível de $1 \%$ de probabilidade de erro, utilizando o software estatístico Estat versão 2 (Estat 1994).

Decorridos 180 dias após a semeadura, foi mensurada a porcentagem de sobrevivência das mudas (Emergência) de acordo com Labouriau \& Valadares (1976), na qual Emergência $(\%)=\mathrm{Ns} / \mathrm{Ni} \times 100$, em que: $\mathrm{Ns}=$ número de sementes semeadas, e $\mathrm{Ni}=$ número de plântulas que emergiram. Também foram mensuradas as seguintes características biométricas de todas as mudas nas seis repetições de cada tratamento: altura da parte aérea $(\mathrm{H})$ e comprimento da raiz (CR), com auxílio de uma régua graduada, expresso em $\mathrm{cm}$ plântula ${ }^{-1}$; diâmetro do colo (DC), com auxílio de paquímetro digital, expresso em $\mathrm{mm}$; número de folhas (NF), computado de modo manual; massa fresca da parte aérea (MFPA), de raiz (MFR) e total (MFT), mensuradas em balança digital, expressos em g plântula ${ }^{-1}$; massas secas da parte aérea (MSPA), de raiz (MSR) e total (MST), com secagem em estufa com circulação de ar a $60^{\circ} \mathrm{C}$, por 72 horas, sendo os resultados expressos em g plântula ${ }^{-1}$. 
Além das características supracitadas, também foi calculado o Índice de Qualidade de Dickson (IQD) de acordo com a proposta de Dickson et al. (1960).

$$
\mathrm{IQD}=\mathrm{MST} /(\mathrm{H} / \mathrm{DC}+(\mathrm{MSPA} / \mathrm{MSR})
$$

Em que: IQD = Índice de Qualidade de Dickson; MST = massa seca total, em g; $\mathrm{H}=$ altura da parte aérea, $\mathrm{em} \mathrm{cm}$; $\mathrm{DC}=$ diâmetro do colo, em mm; MSPA = massa seca da parte aérea, em g; MSR = massa seca da raiz, em gramas.

Com o material vegetal seco em estufa e triturado foram determinados os teores de macro e micronutrientes $\mathrm{N}, \mathrm{P}, \mathrm{K}$, $\mathrm{Ca}, \mathrm{Mg}, \mathrm{S}, \mathrm{Cu}, \mathrm{Zn}, \mathrm{Fe}, \mathrm{Mn}$ e B, além de carbono orgânico na raiz e na parte aérea (folhas e caule) em amostras dos quatro tratamentos (Tedesco et al. 1995, Miyazawa et al. 1999). As análises foram realizadas no Laboratório de Solos na Universidade Federal do Rio Grande do Sul (UFRGS), Porto Alegre, RS.

\section{RESULTADOS E DISCUSSÃO}

A adição de lodo de esgoto promoveu alterações na composição nutricional do substrato dos tratamentos em diferentes magnitudes (Tab. 1).

De modo geral, a adição de lodo de esgoto influenciou positivamente nos atributos de fertilidade do substrato, especialmente nos teores de micronutrientes. Para os macronutrientes houve influência apenas nos teores de N, sendo que para os demais macronutrientes $(\mathrm{Ca}, \mathrm{Mg}, \mathrm{Na} e$ $\mathrm{K})$ houve redução nos teores à medida que aumentaram as proporções de lodo de esgoto. As alterações nos teores de P não foram significativas, tendo-se mantido praticamente inalteradas, em função das doses de lodo de esgoto (Tab. 1).

No que se refere aos micronutrientes analisados nos quatro tratamentos, verificou-se maiores teores nos tratamentos que continham lodo de esgoto (Tab. 1). Os resultados permitem inferir que a presença do lodo de esgoto promoveu maior disponibilidade de $\mathrm{Zn}, \mathrm{Cu}, \mathrm{Fe}$ e Mg na solução do solo, o que certamente contribuiu para maiores teores destes nas mudas. Segundo Taiz \& Zeiger (2017), este resultado pode estar relacionado aos compostos quelados (substâncias orgânicas que envolvem os nutrientes impedindo que sejam ligados a outro elemento, o que poderia causar a sua imobilização), fazendo com que cheguem até a rizosfera para serem absorvidos pelas raízes das plantas.

As características morfológicas e os índices de qualidade das mudas avaliadas no presente estudo apresentaram respostas distintas entre si. Pela análise de variância e teste de Tukey, observou-se diferenças significativas entre as médias dos substratos avaliados para todas as variáveis analisadas, exceto para percentual do crescimento da raiz e percentagem de sobrevivência de plântulas (Tab. 2).

Para as variáveis número de folhas, diâmetro do colo e a altura das mudas, o melhor tratamento foi o T4, o qual apresentou as maiores médias, diferindo estatisticamente de todos os demais. O pior tratamento foi o $\mathrm{T} 1$, o qual apresentou as menores médias, também diferindo estatisticamente de todos os demais (Tab. 2).

Tabela 1. Teores de nutrientes nos substratos utilizados para semeadura de Hovenia dulcis.

\begin{tabular}{|c|c|c|c|c|c|c|c|c|c|c|c|c|}
\hline \multirow{2}{*}{ Trat. } & $\mathrm{N}$ & $\mathrm{Ca}$ & $\mathrm{Mg}$ & $\mathrm{K}$ & $\mathrm{P}$ & $\mathrm{Zn}$ & $\mathrm{Cu}$ & $\mathrm{S}$ & B & $\mathrm{Fe}$ & $\mathrm{Mn}$ & $\mathrm{Na}$ \\
\hline & $\%$ & \multicolumn{4}{|c|}{$\mathrm{cmol}_{\mathrm{c}} \mathrm{L}^{-1}$} & \multicolumn{7}{|c|}{$\mathrm{mg} \mathrm{L}^{-1}$} \\
\hline $\mathrm{T} 1$ & 1,003 & 10,649 & 7,482 & 628 & 383,5 & 24,49 & 0,34 & 79,0 & 0,1 & 2907,5 & 10,87 & 88 \\
\hline $\mathrm{T} 2$ & 1,195 & 12,617 & 7,810 & 564 & 309,0 & 43,72 & 3,61 & 82,2 & 0,1 & 6343,7 & 19,05 & 72 \\
\hline $\mathrm{T} 3$ & 1,371 & 8,406 & 4,678 & 360 & 309,0 & 61,11 & 17,48 & 94,9 & 0,2 & 8697,5 & 18,91 & 44 \\
\hline $\mathrm{T} 4$ & 1,463 & 7,712 & 3,867 & 312 & 309,0 & 61,30 & 22,13 & 87,3 & 0,1 & 10792,4 & 22,18 & 36 \\
\hline
\end{tabular}

Onde T1 (50\% substrato comercial Plantmax ${ }^{\circledR}+50 \%$ cama de equino (composto orgânico), T2 (20\% lodo de esgoto $+80 \%$ composto orgânico), T3 ( $40 \%$ lodo de esgoto $+60 \%$ composto orgânico), $\mathrm{T} 4(60 \%$ lodo de esgoto $+40 \%$ composto orgânico). $\mathrm{N}=$ nitrogênio, Ca $=$ cálcio, $\mathrm{Mg}=$ magnésio, $\mathrm{K}=$ potássio, $\mathrm{P}=$ fósforo, $\mathrm{Zn}=$ zinco, $\mathrm{Cu}=$ cobre, $\mathrm{S}=$ enxofre, $\mathrm{B}=$ boro, $\mathrm{Fe}=$ ferro, $\mathrm{Mn}=$ manganês, $\mathrm{Na}=$ sódio.

Tabela 2. Médias ( \pm desvios padrão) para as variáveis emergência (\%), número de folhas (NF), altura da parte aérea (H), diâmetro do colo (DC), comprimento da raiz (CR), relação altura/diâmetro (H/DC) e Índice de Qualidade de Dickson (IQD) de mudas de Hovenia dulcis em diferentes substratos.

\begin{tabular}{cccccccc}
\hline Trat & E $(\%)$ & NF & DC $(\mathrm{cm})$ & H $(\mathrm{cm})$ & CR & H/DC & IQD \\
\hline T1 & $100 \pm 0,00 \mathrm{a}$ & $4,96 \pm 0,16 \mathrm{c}$ & $1,23 \pm 0,00 \mathrm{c}$ & $4,92 \pm 0,17 \mathrm{~d}$ & $19,58 \pm 0,34 \mathrm{a}$ & $4,00 \pm 0,15 \mathrm{~d}$ & $0,51 \pm 0,01 \mathrm{~d}$ \\
T2 & $100 \pm 0,00 \mathrm{a}$ & $9,56 \pm 0,38 \mathrm{~b}$ & $1,69 \pm 0,03 \mathrm{~b}$ & $9,65 \pm 0,29 \mathrm{c}$ & $19,40 \pm 0,45 \mathrm{a}$ & $5,69 \pm 0,12 \mathrm{c}$ & $1,21 \pm 0,07 \mathrm{~b}$ \\
T3 & $100 \pm 0,00 \mathrm{a}$ & $9,96 \pm 0,07 \mathrm{~b}$ & $1,69 \pm 0,01 \mathrm{~b}$ & $16,14 \pm 0,67 \mathrm{~b}$ & $19,79 \pm 0,25 \mathrm{a}$ & $9,54 \pm 0,33 \mathrm{~b}$ & $1,06 \pm 0,08 \mathrm{c}$ \\
T4 & $100 \pm 0,00 \mathrm{a}$ & $12,25 \pm 0,31 \mathrm{a}$ & $1,99 \pm 0,06 \mathrm{a}$ & $22,87 \pm 0,52 \mathrm{a}$ & $19,56 \pm 0,21 \mathrm{a}$ & $11,50 \pm 0,55 \mathrm{a}$ & $1,41 \pm 0,08 \mathrm{a}$ \\
\hline
\end{tabular}

Onde T1 (50\% substrato comercial Plantmax ${ }^{\circledR}+50 \%$ cama de equino) (composto orgânico), T2 (20\% lodo de esgoto + 80\% composto orgânico), T3 (40\% lodo de esgoto $+60 \%$ composto orgânico), T4 (60\% lodo de esgoto $+40 \%$ composto orgânico). Médias seguidas pelas mesmas letras nas colunas não diferem significativamente pelo teste Tukey a $1 \%$ de probabilidade. 
O maior número de folhas está totalmente associado ao desenvolvimento da planta, já que é o local fundamental para que ocorra a fotossíntese e por serem centros de reserva, fonte de fitormônios e cofatores de enraizamento que são transportados para a base, auxiliando na formação de novos tecidos, como as raízes (Taiz \& Zeiger 2017).

Resultados semelhantes aos encontrados no presente estudo foram obtidos por Delarmelina et al. (2013) na produção de mudas de cambaí-amarelo (Sesbania virgata (Cav.) Pers), Marques et al. (2018), trabalhando com produção e qualidade de mudas de araçá-vermelho (Psidium cattleianum var. cattleianum Sabine) e Santos et al. (2019) utilizando as mesmas proporções de lodo de esgoto na produção e qualidade de mudas de angicovermelho (Parapiptadenia rigida (Benth.) Brenan.). Nestes estudos foram observados os maiores valores para número de folhas, diâmetro do colo e altura das mudas produzidas nas combinações de $40 \% \mathrm{LE}+60 \% \mathrm{CO}$ e $60 \% \mathrm{LE}+40 \%$ $\mathrm{CO}$, os mesmos tratamentos utilizados no presente estudo.

Para obter mudas de espécies florestais de boa qualidade, são recomendados limites de altura entre 20 e $35 \mathrm{~cm}$ (Gonçalves et al. 2014). Seguindo a variação de qualidade apresentada, os tratamentos testados neste trabalho compostos com lodo de esgoto se estabeleceram dentro do padrão de qualidade recomendado. $\mathrm{O}$ tratamento testemunha (T1) apresentou a menor média, $4,92 \mathrm{~cm}$, bastante inferior aos tratamentos com presença de lodo de esgoto e, as mudas no tratamento T4 alcançaram altura média de $22,87 \mathrm{~cm}$. Ainda, todos os tratamentos formulados com lodo de esgoto apresentaram médias significativamente maiores para a altura de mudas quando comparado ao tratamento testemunha (T1) (Tab. 2). Segundo Caldeira et al. (2014), a altura da parte aérea das mudas demonstra uma ótima estimativa do crescimento inicial da muda no campo, sendo tecnicamente, aceita como uma boa medida do potencial de desempenho.

Os tratamentos com adição de lodo de esgoto apresentaram maiores médias para o diâmetro do colo das mudas de H. dulcis, quando comparado ao tratamento T1 (testemunha) (Tab. 2). Daniel et al. (1997) consideram esse parâmetro como um dos mais importantes para avaliar a sobrevivência logo após o plantio de mudas de diferentes espécies florestais. A avaliação do diâmetro do colo, frequentemente, é recomendada para indicar a capacidade de sobrevivência das mudas no campo.

As mudas produzidas no tratamento que continha $60 \%$ de lodo de esgoto (T4) apresentou maior média de altura e, como consequência também foi verificado o maior número de folhas, que são responsáveis pelo metabolismo primário e secundário, induzindo maior crescimento e maior diâmetro do colo e, como relatado por alguns pesquisadores, estes parâmetros favorecem a qualidade das mudas para plantio a campo.

Para a relação entre altura e diâmetro do colo (H/DC) o melhor tratamento foi o tratamento T4 $(60 \% \mathrm{LE}+40 \%$ $\mathrm{CO}$ ) com média de 11,50, diferindo de todos os demais. O pior tratamento também foi o T1, diferindo de todos os demais (Tab. 2). Cabe ressaltar que, esta característica varia conforme a espécie, o tipo e proporção do substrato, do volume do recipiente, do manejo das mudas no viveiro e da idade em que a muda foi analisada (Trazzi et al. 2013; Caldeira et al. 2014). Constatou-se em estudos com espécies florestais que mudas com maior altura e maior diâmetro do colo demonstraram maior potencial de crescimento inicial no campo (Trigueiro \& Guerrini 2003, Caldeira et al. 2012, Marques et al. 2018, Santos et al. 2019).

Outro parâmetro avaliado que evidencia a qualidade das mudas e foi influenciada pelos diferentes tratamentos foi o Î́ndice de Qualidade de Dickson (IQD), que na sua interpretação considera a robustez e o equilíbrio da distribuição da biomassa na muda, considerando os resultados de vários parâmetros importantes, utilizados na avaliação da qualidade das mudas (Fonseca et al. 2002).

Considerando o valor mínimo do IQD de 0,20 (Hunt 1990), os resultados demonstram que as mudas de $H$. dulcis, oriundas de todos os tratamentos atingiram valores superiores, indicando qualidade para serem transplantadas no campo, especialmente dos tratamentos formulados com adição de lodo de esgoto e, sendo observados melhores resultados desta variável no tratamento T4 $(60 \% \mathrm{LE}+40 \%$ $\mathrm{CO}$ ), o qual diferiu estatisticamente de todos os demais (Tab. 2). Caldeira et al. (2012) relatam que quanto maior o valor do IQD, melhor será o padrão de qualidade obtido com as mudas. Tendo como base essa afirmação, podemos considerar que as mudas produzidas no tratamento $\mathrm{T} 4$ são as de melhor qualidade e que provavelmente melhor se adaptarão quando plantadas no campo. Adicionalmente, Marques et al. (2018) e Santos et al. (2019) também avaliaram este parâmetro na produção de mudas de araçá-vermelho e angico-vermelho, respectivamente e, encontraram maiores valores quando utilizaram substratos formulados com lodo de esgoto.

Ainda, alguns trabalhos enfatizam que o IQD é uma característica variável. É possível observar que este índice pode variar em função da espécie, do manejo das mudas no viveiro, do tipo e proporção do substrato, do volume do recipiente e, principalmente, com a idade em que a muda foi avaliada (Saidelles et al. 2009, Trazzi et al. 2013, Caldeira et al. 2014).

Para o comprimento da raiz não foram observadas diferenças significativas entre as médias dos tratamentos (Tab. 2), possivelmente o tamanho e a forma do recipiente utilizado tenham influência sobre essa resposta, tornando limitado o potencial de desenvolvimento das raízes. Entretanto, diferenças significativas foram detectadas para a média da biomassa dessa estrutura vegetativa tanto para a massa fresca como para a massa seca (Tab. 3). Essa discrepância pode ser visualmente observada, pois os tratamentos T2, T3 e T4 que possuem lodo de esgoto, produziram grande quantidade de raízes secundárias se comparadas ao tratamento testemunha (T1).

De modo geral, o emprego do lodo de esgoto na composição dos substratos fez com que houvessem efeitos relevantes na biomassa das mudas, com aumento da biomassa fresca, seca e total das mudas de H. dulcis (Tab. 3). 
Os tratamentos que continham lodo de esgoto na sua composição apresentaram maiores massas de raiz, tanto fresca como seca $\left(66,344\right.$ e 8,060 g planta ${ }^{-1}$, respectivamente), sendo que o T4 ( $60 \%$ de lodo de esgoto) se mostrando como o melhor tratamento (Tab. 3). Este resultado é devido, possivelmente, a presença de matéria orgânica, porosidade, aeração e baixa densidade, concedendo pouca resistência ao crescimento radicular (Delarmelina et al. 2013).

Vários trabalhos com espécies florestais têm reconhecido que a massa seca de raízes como uma das melhores características para se avaliar a sobrevivência e o crescimento inicial das mudas no campo (Caldeira et al. 2012, Caldeira et al. 2014). O aumento significativo na biomassa das raízes nos tratamentos formulados com lodo de esgoto é decorrente do acréscimo no maior número de raízes laterais nesses tratamentos (Trazzi et al. 2014).

Da mesma forma que foi observado para biomassa do sistema radicular, constatou-se, resultados semelhantes quanto a massa fresca, seca e total da parte aérea $(33,679$, 9,871 e 17,931 g planta $^{-1}$, respectivamente) (Tab. 3). As maiores médias de biomassa da parte aérea foram observadas no tratamento $\mathrm{T} 4(60 \% \mathrm{LE}+40 \% \mathrm{CO})$ e, as menores médias para biomassa da parte aérea foram verificadas no tratamento T1 (Tab. 3), sendo que todas diferiram entre si. Adicionalmente, foi observado que a biomassa da raiz e da parte aérea se mostrou responsiva à proporção de lodo de esgoto adicionado ao composto orgânico, ou seja, as maiores médias foram observadas no tratamento $\mathrm{T} 4$, seguido do tratamento $\mathrm{T} 3 \mathrm{e}$, do tratamento $\mathrm{T} 2$, sendo que todos apresentaram diferença significativa entre si (Tab. 3).

Trigueiro \& Guerrini (2003) analisando as características físicas e químicas de substratos com diferentes proporções de lodo de esgoto e casca de arroz carbonizada, relataram que quando adicionado mais lodo de esgoto no substrato, ocorre também o aumento da densidade e do percentual de microporos e, assim sendo, acontece a elevação da capacidade de reter água. Alguns substratos leves e de baixa densidade, como fibra de coco, vermiculita e casca de arroz, aumentam a macroporosidade das misturas diminuindo a capacidade de retenção de água do substrato (Gonçalves et al. 2014). Assim, cada espécie responderá de forma diferente em relação ao crescimento e biomassa da raiz, tendo influência à proporção e materiais utilizados, recipiente acondicionante, dentre outros. Vale ressaltar que no tratamento $\mathrm{T} 4$ também foram encontrados maiores número de folhas, altura da parte aérea e diâmetro do colo; assim, corroborando para o maior resultado de biomassa nessa estrutura vegetativa (Tabs. $2 \mathrm{e} 3$ ) e, consequentemente, as mudas responderam com considerável elevação de macro e micronutrientes acumulados tanto na raiz quanto na parte aérea (Tab. 4).

Tabela 3. Médias ( \pm desvios padrão) para as variáveis massa fresca radicular (MFR), massa fresca da parte aérea (MFPA), massa fresca total (MFT), massa seca radicular (MSR), massa seca da parte aérea (MSPA), massa seca total (MST), de mudas de Hovenia dulcis em diferentes substratos.

\begin{tabular}{ccccccc}
\hline \multirow{2}{*}{ Trat. } & \multicolumn{1}{c}{ MFR } & MFPA & MFT & MSR & MSPA & MST \\
\cline { 2 - 7 } & \multicolumn{7}{c}{ g plântula $^{-1}$} \\
\hline T1 & $11,102 \pm 0,271 \mathrm{~d}$ & $3,511 \pm 0,088 \mathrm{~d}$ & $14,613 \pm 0,237 \mathrm{~d}$ & $1,348 \pm 0,058 \mathrm{~d}$ & $1,164 \pm 0,035 \mathrm{~d}$ & $2,512 \pm 0,058 \mathrm{~d}$ \\
T2 & $37,226 \pm 2,870 \mathrm{c}$ & $14,384 \pm 0,910 \mathrm{c}$ & $51,611 \pm 3,327 \mathrm{c}$ & $4,055 \pm 0,345 \mathrm{c}$ & $4,090 \pm 0,218 \mathrm{c}$ & $8,145 \pm 0,495 \mathrm{c}$ \\
T3 & $42,545 \pm 2,922 \mathrm{~b}$ & $22,222 \pm 1,674 \mathrm{~b}$ & $64,767 \pm 2,650 \mathrm{~b}$ & $5,222 \pm 0,860 \mathrm{~b}$ & $6,175 \pm 0,502 \mathrm{~b}$ & $11,398 \pm 1,123 \mathrm{~b}$ \\
T4 & $66,344 \pm 1,953 \mathrm{a}$ & $33,679 \pm 1,671 \mathrm{a}$ & $100,024 \pm 2,882 \mathrm{a}$ & $8,060 \pm 0,468 \mathrm{a}$ & $9,871 \pm 0,580 \mathrm{a}$ & $17,931 \pm 0,965 \mathrm{a}$ \\
\hline
\end{tabular}

Onde T1 (50\% substrato comercial Plantmax $®+50 \%$ cama de equino) (composto orgânico), T2 (20\% lodo de esgoto + $80 \%$ composto orgânico), T3 (40\% lodo de esgoto $+60 \%$ composto orgânico), T4 (60\% lodo de esgoto $+40 \%$ composto orgânico). Médias seguidas pelas mesmas letras nas colunas não diferem significativamente pelo teste Tukey a $1 \%$ de probabilidade.

Tabela 4. Teores de nutrientes no sistema radicular e parte aérea de mudas de Hovenia dulcis nos diferentes substratos

\begin{tabular}{|c|c|c|c|c|c|c|c|c|c|c|c|c|}
\hline \multirow{2}{*}{\multicolumn{2}{|c|}{ Tratamento }} & $\mathrm{N}$ & $\mathrm{P}$ & $\mathrm{K}$ & $\mathrm{Ca}$ & $\mathrm{Mg}$ & $\mathrm{S}$ & $\mathrm{Cu}$ & $\mathrm{Zn}$ & $\mathrm{Fe}$ & $\mathrm{Mn}$ & B \\
\hline & & \multicolumn{6}{|c|}{$\mathrm{g} \mathrm{Kg}^{-1}$} & \multicolumn{5}{|c|}{$\mathrm{mg} \mathrm{Kg}^{-1}$} \\
\hline \multirow{4}{*}{ Raiz } & $\mathrm{T} 1$ & 6,9 & 2,6 & 25,0 & 4,1 & 6,8 & 2,1 & 5 & 321 & 395 & 161 & 26 \\
\hline & $\mathrm{T} 2$ & 9,9 & 2,9 & 18,0 & 4,1 & 7,8 & 2,2 & 8 & 535 & 673 & 295 & 16 \\
\hline & $\mathrm{T} 3$ & 11,0 & 2,6 & 12,0 & 4,3 & 7,8 & 2,7 & 10 & 1200 & 997 & 372 & 16 \\
\hline & $\mathrm{T} 4$ & 14,0 & 2,3 & 6,0 & 3,5 & 8,0 & 2,7 & 14 & 1100 & 1500 & 584 & 14 \\
\hline \multirow{4}{*}{$\begin{array}{l}\text { Parte } \\
\text { aérea }\end{array}$} & $\mathrm{T} 1$ & 9,2 & 5,0 & 17,7 & 13,0 & 3,5 & 1,3 & 7 & 16 & 913 & 54 & 16 \\
\hline & $\mathrm{T} 2$ & 11,0 & 5,4 & 10,0 & 15,0 & 3,2 & 0,8 & 3 & 47 & 200 & 166 & 28 \\
\hline & $\mathrm{T} 3$ & 13,0 & 6,2 & 9,5 & 17,0 & 3,3 & 0,8 & 3 & 92 & 176 & 381 & 28 \\
\hline & $\mathrm{T} 4$ & 14,0 & 7,5 & 7,2 & 19,0 & 3,3 & 0,8 & 3 & 124 & 119 & 425 & 32 \\
\hline
\end{tabular}

T1 (50\% substrato comercial Plantmax ${ }^{\circledR}+50 \%$ cama de equino) (composto orgânico), T2 (20\% lodo de esgoto + $80 \%$ composto orgânico), T3 (40\% lodo de esgoto $+60 \%$ composto orgânico), T4 (60\% lodo de esgoto $+40 \%$ composto orgânico). $\mathrm{N}=$ nitrogênio, $\mathrm{P}=$ fósforo, $\mathrm{K}=$ potássio, $\mathrm{Ca}=$ cálcio, $\mathrm{Mg}$ = magnésio, $\mathrm{S}=$ enxofre, $\mathrm{Cu}=$ cobre, $\mathrm{Zn}$ $=$ zinco, $\mathrm{Fe}=$ ferro, $\mathrm{Mn}=$ manganês, $\mathrm{B}=$ boro . 
$\mathrm{Na}$ raiz, os teores de $\mathrm{N}, \mathrm{Mg}$ e $\mathrm{S}$ aumentaram significativamente com adição de lodo de esgoto ao composto orgânico e, os teores de $\mathrm{P}, \mathrm{K}$ e $\mathrm{Ca}$ foram reduzidos nos tratamentos com presença crescente de lodo de esgoto (Tab. 4). Diferentemente, na parte aérea, os teores de N, P e Ca aumentaram nos tratamentos com adição de lodo de esgoto e, houve redução acentuada para o teor de K; já para os teores de $\mathrm{Mg}$ e de S não foram observadas alterações com adição das diferentes proporções do lodo de esgoto (Tab. 4). Araújo et al. (2009) avaliando o efeito de doses de lodo de esgoto na nutrição de Brachiaria decumbens Stapf, observaram que nos tratamentos com lodo de esgoto os teores de $\mathrm{P}$ aumentaram gradativamente mas, mesmo na maior dose de lodo, o teor foliar de P não diferiu significativamente da testemunha.

Adicionalmente, a tendência geral da ordem crescente do teor dos macronutrientes avaliados na raiz foi de $\mathrm{K}>\mathrm{N}$ $>\mathrm{Mg}>\mathrm{Ca}>\mathrm{P}>\mathrm{S}$ e na parte aérea $\mathrm{Ca}>\mathrm{N}>\mathrm{K}>\mathrm{P}>\mathrm{Mg}$ $>\mathrm{S}$, sendo que, tanto na raiz como na parte aérea, o $\mathrm{S}$ foi encontrado em menor concentração (Tab. 4). Camargo et al. (2013) analisaram o teor de macronutrientes em folhas de pinhão-manso (Jatropha curcas L.), cultivadas durante 60 dias, e verificaram que $\mathrm{Ne} \mathrm{S}$ foram detectados em maior e menor concentração, respectivamente, segundo a ordem $\mathrm{N}>$ $\mathrm{K}>\mathrm{Mg}>\mathrm{Ca}>\mathrm{P}>\mathrm{S}$. Já Santos et al. (2019) em seu trabalho com angico-vermelho e nas mesmas proporções de lodo de esgoto, encontraram nas folhas a mesma concentração de macronutrientes do presente estudo e, para a raiz, teor nutricional semelhante, sendo a ordem $\mathrm{N}>\mathrm{K}>\mathrm{Mg}>\mathrm{Ca}$ $>\mathrm{P}>\mathrm{S}$. No presente estudo, o K esteve mais concentrado na raiz, já na parte aérea foi o $\mathrm{Ca}$ (Tab. 4).

Os teores de $\mathrm{Cu}, \mathrm{Zn}, \mathrm{Fe}$ e $\mathrm{Mn}$ na raiz aumentaram significativamente com a adição das diferentes proporções de lodo de esgoto, e o teor de B diminuiu. Entretanto, para a parte aérea, os teores desse micronutriente juntamente com Zn e Mn, aumentaram gradativamente nos tratamentos com adição de lodo de esgoto e, para $\mathrm{Cu}$ e Fe, foi observada redução à medida que foi adicionado maiores proporções de lodo de esgoto (Tab. 4).

Adicionalmente, a ordem geral e crescente do teor dos micronutrientes avaliados na raiz foi de $\mathrm{Fe}>\mathrm{Zn}>\mathrm{Mn}>\mathrm{B}>$ $\mathrm{Cu}$ e na parte aérea foi de $\mathrm{Fe}>\mathrm{Mn}>\mathrm{Zn}>\mathrm{B}>\mathrm{Cu}$ (Tab. 4). Nota-se que nas duas estruturas vegetativas analisadas, houve altos teores de $\mathrm{Fe}$ e $\mathrm{Zn}$ e baixos de $\mathrm{Cu}$ (Tab. 4).

Santos et al. (2019), em seu trabalho com angicovermelho observaram no sistema radicular, aumento nos teores de $\mathrm{Cu}, \mathrm{Zn}, \mathrm{Fe}$ e, Mn com a adição do lodo de esgoto; já na parte aérea este comportamento foi observado apenas para $\mathrm{Zn}$ e $\mathrm{Mn}$; para $\mathrm{Cu}$ e B não foram observadas alterações marcantes nos teores e, para o Fe houve redução na sua concentração com a adição do lodo de esgoto. Toledo et al. (2013) avaliando a qualidade química de substratos obtidos a partir da combinação de lodo de esgoto ao substrato comercial e, os teores nutricionais das folhas do híbrido Eucalyptus urograndis, verificaram que os teores de $\mathrm{Zn}$, $\mathrm{Fe}, \mathrm{Mn}$ e B aumentaram com a adição de lodo de esgoto ao substrato e, como evidenciado no presente estudo, o
$\mathrm{Cu}$ foi o único que não teve sua concentração alterada em função da adição de lodo de esgoto. Esse fato pode ser explicado pelo valor de $\mathrm{pH}$ do substrato (acidez), que diminuiu a disponibilidade desse nutriente para a planta (Toledo et al. 2013).

Segundo Antunes et al. (2016), a maior concentração de alguns micronutrientes nas partes vegetativas quando há a adição de lodos biológicos, pode ser advinda de disponibilização destes nutrientes, pois estes tratamentos apresentam concentrações elevadas dos mesmos. Vale ressaltar que, podem ser encontrados resultados conflitantes com a literatura quanto à dinâmica de acumulação de nutrientes em tecidos de plantas, em função da aplicação de lodo de esgoto. Todavia, há de se considerar a possibilidade do efeito de muitos fatores como a composição química do lodo, o período entre a aplicação no solo e as coletas de tecidos para análises, as características da espécie vegetal estudada e as possíveis interações com outros fatores. Ainda, deve-se levar em consideração que o lodo de esgoto não é um produto com composição química e características padronizadas, podendo ter suas características influenciadas pelos detalhes do processo de compostagem e pelas características dos resíduos utilizados (Caldeira et al. 2014).

Diversos pesquisadores testaram lodo de esgoto nas mais diferentes concentrações e com várias espécies arbóreas florestais nativas, exóticas e também frutíferas (Prates et al. 2011). Constatou-se que o lodo de esgoto apresenta excelentes resultados, mostrando efeitos benéficos em seu uso como parte da composição de substratos, como mostrado em alguns trabalhos na literatura (Faustino et al. 2005, Caldeira et al. 2012, Delarmelina et al. 2013, Marques et al. 2018, Santos et al. 2019).

Devido aos efeitos benéficos da adição de resíduos orgânicos, tais como a melhoria da atividade biológica, condicionamento do solo, estabilidade física, menor taxa de mineralização, dentre outros, vários estudos na área de produção de mudas afirmam que a adubação orgânica se mostra como uma adubação mais interessante ao solo e meio ambiente. Ainda, por meio dos resultados obtidos no presente estudo e da literatura, pode-se observar que o lodo de esgoto é uma matéria-prima interessante para compor substrato para a produção de mudas de espécies florestais.

\section{REFERÊNCIAS}

Antunes, R.M., Castilhos, R.M.V., Castilho, D.D., Leal, O.A. dos \& Andreazza, R. 2016. Crescimento inicial de acácia-negra com vermicompostos de diferentes resíduos agroindustriais. Ciência Florestal 26(1):1-9.

Araújo, F.F., Gil, F.C. \& Tiritan, C.S. 2009. Lodo de esgoto na fertilidade do solo, na nutrição de Brachiaria decumbens e na atividade da desidrogenase. Pesquisa Agropecuária Tropical 9(1):1-6.

Caldeira, M.V.W., Peroni, L., Gomes, D.R., Delarmelina, W.M. \& Trazzi, P.A. 2012. Diferentes proporções de biossólido na composição de substratos para a produção de mudas de timbó (Ateleia glazioveana Baill). Scientia Forestalis 40:15-22.

Caldeira, M.V.W., Favalessa, M., Gonçalves E. de O., Delarmelina, W.M., Santos, F.E.V. \& Vieira, M. 2014. Lodo de esgoto como componente de substrato para produção de mudas de Acacia mangium Willd. Comunicata Scientiae 5(1):34-43. 
Camargo, R. de, Maldonado, A.C.D., Dias, P.A.S., Souza, M.F. \& França, M.S. 2013. Diagnose foliar em mudas de pinhão-manso (Jatropha curcas L.) produzidas com biossólido. Revista Brasileira de Engenharia Agrícola e Ambiental 17(3):283-290.

Cruz, C.A.F., Paiva, H.N., Gomes, K.C.O. \& Guerrero, C.R.A. 2004. Efeito de diferentes níveis de saturação por bases no desenvolvimento e qualidade de mudas de ipê roxo (Tabebuia impetiginosa (Mart.) Standley). Scientia Forestalis 66:100-107.

Daniel, O., Vitorino, A.C.T., Aloisi, A.A., Mazzochin, L., Tokura, A.M., Pinheiro, E.R. \& Souza, E.F. 1997. Aplicação de fósforo em mudas de Acacia mangium. Revista Árvore 21(2):163-168.

Delarmelina, W.M., Caldeira, M.V.W., Faria, J.C.T. \& Gonçalves, E.O. 2013. Uso de lodo de esgoto e resíduos orgânicos no crescimento de mudas de Sesbania virgata (Cav.) Pers. Revista Agroambiente 7(1):184-192.

Dickson, A.; Leaf, A.L. \& Hosner, J.F. 1960. Quality appraisal of white spruce and white pine seedling stock in nurseries. Forestry Chronicle 36(1):10-13.

Estat. (ESTAT 2.0). 1994. Sistema de Análise Estatística. Pólo Computacional do Departamento de Ciências Exatas da UNESP, Jaboticabal.

Faustino, R., Kato, M.T., Flôrencio, L. \& Gavazza, S. 2005. Lodo de esgoto como substrato na produção de Senna siamea Lam. Revista Brasileira de Engenharia Agrícola e Ambiental 9:278-282.

Ferraz, A. de V., Poggiani, F. \& Silva, P.H.M. da. 2016. Aplicação de lodo de esgoto seco e fertilizantes minerais em plantios de Pinus caribaea var. hondurensis: produtividade e balanço de nutrientes. Scientia Forestalis 44(112):959-970.

Fonseca, E.P. de, Valéri, S.V., Miglioranza, E., Fonseca, N.A.N. \& Couto, L. 2002. Padrão de qualidade de mudas de Trema micrantha (L.) Blume, produzidas sob diferentes períodos de sombreamento. Revista Árvore 26(4):515-523.

Gonçalves, E.O., Petri, G.M., Caldeira, M.V.W., Dalmaso, T.T. \& Silva, A.G. 2014. Crescimento de mudas de Ateleia glazioviana em substratos contendo diferentes materiais orgânicos. Floresta e Ambiente 21(3):339-348.

Hunt, G.A. 1990. Effect of styroblock design and cooper treatment on morphology of conifer seedlings. In: Target Seedlings Symposium: Proceedings, Combined Meeting of the Western Forest Nursery Associations (1990 August 13-17). United States Department of Agriculture, Forest Service, Fort Collins, 218-222 p.

Labouriau, L.F.G. \& Valadares, M.E.B. 1976. On the germination of seeds of Calotropis procera. Anais da Academia Brasileira de Ciências 48(2):263-184

Marques, A.R.F., Oliveira, V. da S., Boligon, A.A. \& Vestena, S. 2018. Produção e qualidade de mudas de Psidium cattleianum var. cattleianum Sabine (Myrtaceae) em diferentes substratos. Acta Biológica Catarinense 5(1):5-13.

Miyazawa, M., Pavan, M.A. \& Muraoka, T. 1999. Análises químicas de tecido vegetal. In: Manual de análises químicas de solo, plantas e fertilizantes. Silva, F. C. (Org). Embrapa Comunicação para Transferência de Tecnologia, Brasília, p. 171-224.

Noernberg, S. 2009. Avaliação e quantificação da regeneração de Hovenia dulcis em um remanescente de Floresta Ombrófila Mista. Trabalho de conclusão de curso. 79 f., Universidade do Contestado, Canoinhas.

Paiva, A.V. de, Poggiani, F., Gonçalves, J.L. de M. \& Ferraz, A. de V. 2009. Crescimento de mudas de espécies arbóreas nativas, adubadas com diferentes doses de lodo de esgoto seco e com fertilização mineral. Scientia Forestalis 37(84):499-511.

Prates, F.B. de S., Sampaio, R.A., Silva, W.J. da, Fernandes, L.A., Junio, G.R.Z. \& Saturnino, H.M. 2011. Crescimento e teores de macronutrientes em pinha-manso adubado com lodo de esgoto e silicato de cálcio e magnésio. Revista Caatinga 24(2):101-112.

Ribeiro, V. de M., Valmorbida, R., Hartmann, K.C.D., Porto, E.C., Almeida, J., Corsato, J.M. \& Fortes, A.M.T. 2019. Efeito alelopático de Leucaena leucocephala e Hovenia dulcis sobre germinação de Mimosa bimucronata e Peltophorum dubium. Iheringia: Série Botânica 74:1-7.

Rosa, S.F., Longhi, S.J. \& Ludwig, M.P. 2008. Aspectos florísticos e fitossociológicos da Reserva Capão de Tupanciretã, Tupanciretã, RS. Ciência Florestal 18(1):15-25.

Saidelles, F.L.F., Caldeira, M.V.W., Schirmer, W.N. \& Sperandio, H.V. 2009. Casca de arroz carbonizada como substrato para produção de mudas de tamboril-da-mata e garapeira. Semina: Ciências Agrárias 30(1):173-186.

Santos, F.E.V., Caldeira, M.V.W. \& Kunz, S.H. 2013. Qualidade de mudas de Parapiptadenia rigida (Benth.) Brenan produzidas em diferentes substratos com lodo de esgoto e casca de arroz. Ecologia e Nutrição Florestal 1(3):55-62.

Santos, R.P. dos, Costa, A.L., Pedroso, L.D., Travessas, A.O. \& Vestena, S. 2019. Efeito do lodo de esgoto na produção e nutrição de mudas de angico-vermelho (Parapiptadenia rigida (Benth.) Brenan). Ecologia e Nutrição Florestal 7(8):1-10.

Scheer, M.B., Carneiro, C. \& Santos, K.G. dos. 2010. Substratos à base de lodo de esgoto compostado na produção de mudas de Parapiptadenia rigida (Benth.) Brenan. Scientia Forestalis 38(8):637-644.

Taiz, L. \& Zeiger, E. 2017. Fisiologia do desenvolvimento vegetal. Artmed, Porto Alegre. 820 p.

Tedesco, M.J. Gianello, C., Bissani, C. A., Bohnen, H. \& Volkweiss, S. J. 1995. Análise de solos, plantas e outros materiais. UFRGS (Boletim Técnico), Porto Alegre. $174 \mathrm{p}$.

Toledo, F.H.S.F. de, Venturin, N., Macedo, R.L.G., Dias, B.A.S., Silva, I.M.M. da; Neves, Y.Y.B., Nascimento, G. de O. \& Carlos, L. 2013. Influência da qualidade química do substrato no teor de nutrientes em folhas de mudas de eucalipto. Ecologia e Nutrição Florestal 1(2):89-96.

Trazzi, P.A., Caldeira, M.V.W., Passos, R.R. \& Gonçalves, E.O. 2013. Substratos de origem orgânica para produção de mudas de teca (Tectona grandis Linn. F.). Ciência Florestal 23(3):401-409.

Trazzi, P.A., Delarmelina, W.M. Caldeira \& M.V.W. 2014. Concentração e quantidade de nutrientes em mudas de Teca produzidas em substratos orgânicos. Ecologia e Nutrição Florestal 2(1):19-31.

Trigueiro, R. de M. \& Guerrini, I.A. 2003. Uso de biossólidos como substratos para produção de mudas de eucalipto. Scientia Forestalis 64(2): $150-1625$.

Wandscheer, A.C.D., Borella, J., Bonatti, L.C. \& Pastorini L.H. 2011. Atividade alelopática de folhas e pseudofrutos de Hovenia dulcis Thunb. (Rhamnaceae) sobre a germinação de Lactuca sativa $\mathrm{L}$. (Asteraceae). Acta Botanica Brasilica 25(1):25-30.

Zhou, Y., Newman, C., Xie, Z. \& Macdonald, D.W. 2013. Peduncles elicit large-mammal endozoochory in a dry-fruited plant. Annals of Botany 112(1):85-93. 